FERMILAB-TM-2530-APC

\title{
Mysterious Dipole Synchrotron Oscillations During and After Adiabatic Capture*
}

\author{
K.Y. Ng
}

(March 2012)

\begin{abstract}
Strong synchrotron oscillations were observed during and after the 2.5- $\mathrm{MHz}$ rf adiabatic capture of a debunched booster batch in the Main Injector. Analysis shows two possible sources for the synchrotron oscillations. One is the frequency drift of the $2.5-\mathrm{MHz}$ rf after the turning off of the $53-\mathrm{MHz}$ rf voltage, thus resulting in an energy mismatch with the debunched beam. The second source is the energy mismatch of the injected booster beam with the frequency of the $53-\mathrm{MHz} \mathrm{rf}$. We have been able to rule out the first possibility.
\end{abstract}

*Work supported by Fermi Research Alliance, LLC under Contract No. DE-AC02-07CH11359 with the US Department of Energy. 


\section{Introduction}

Strong dipole synchrotron oscillations with amplitude about $\Delta \tau \approx 15$ ns were observed in the Main Injector during and after adiabatic capture with the 2.5-MHz rf from a debunched beam destined for the $g-2$ experiment (see Fig. 1). Simulations of adiabatic capture, however, usually show right-left symmetry of the linear density. As a result, one is curious about where these synchrotron oscillations come from. A preliminary guess is that the energy of the beam does not match the frequency of the capturing rf. It is the purpose of this note to compute the necessary energy mismatch to produce the observable synchrotron oscillations and to determine whether such mismatch is possible.

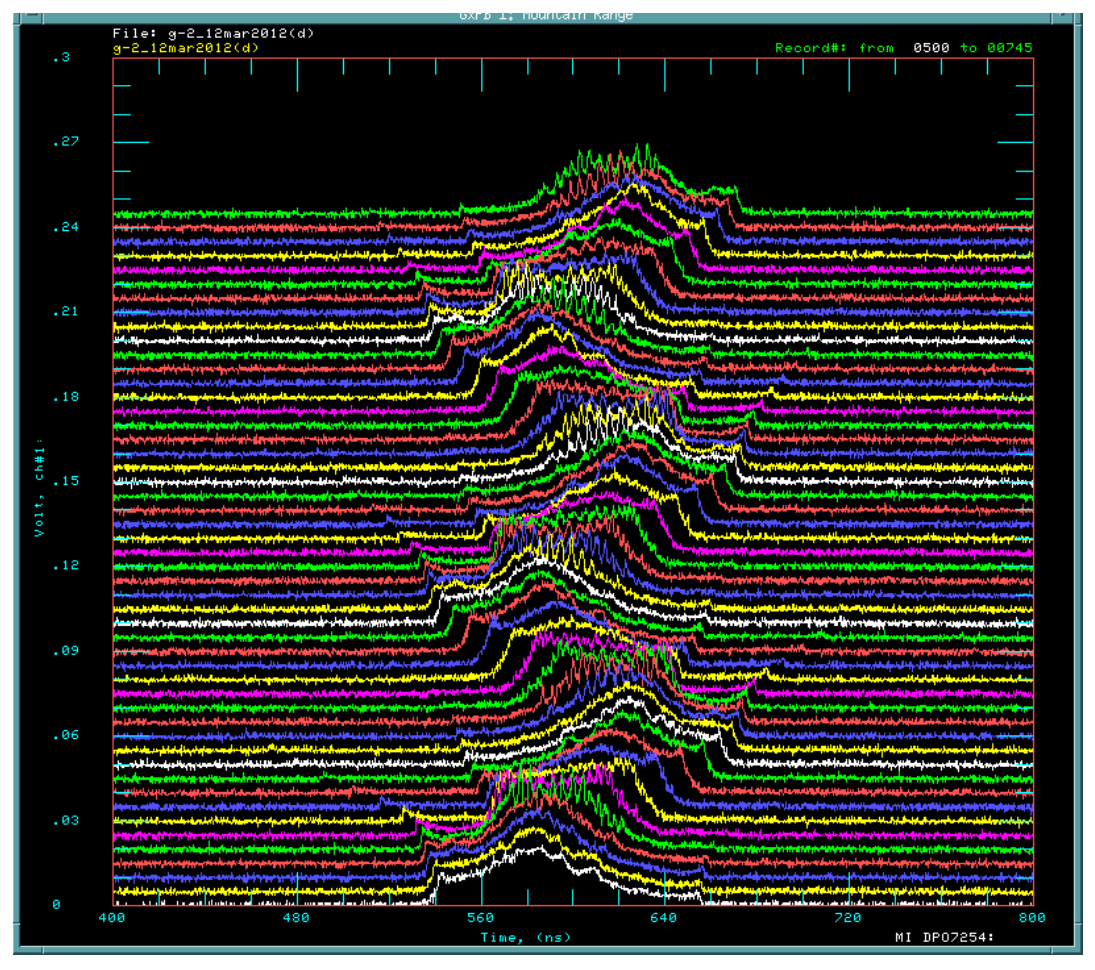

Figure 1: The second of four bunches captured by $2.5 \mathrm{MHz}$ rf from 0.5 to $65 \mathrm{kV}$ in $90 \mathrm{~ms}$. Strong synchrotron oscillation was observed during the capture with oscillation amplitude $\Delta \tau \approx 15 \mathrm{~ns}$. This study was made on March 12, 2012. Although the maximum rf voltage was set at $75 \mathrm{kV}$ during the study, we observed that the voltage actually got up to about $70 \mathrm{kV}$ and then drifted down to $65 \mathrm{kV}$. 


\section{Energy Mismatch}

With $2.5 \mathrm{MHz}$ rf at roughly $65 \mathrm{kV}$, we want to compute the frequency offset that can lead to a synchrotron oscillation of amplitude about 15 ns. Some properties of the Main Injector are listed in Table I, of which the first 4 lines are input.

Table I: Some properties of the Main Injector.

\begin{tabular}{lrl}
\hline Ring circumference $C$ & 3319.42 & $\mathrm{~m}$ \\
Nominal particle energy $E$ & 8.9393 & $\mathrm{GeV}$ \\
2.5MHz rf harmonic $h$ & 28 & \\
Slip factor $\eta$ & $-8.18 \times 10^{-3}$ & \\
gamma $\gamma$ & 9.52631 & \\
beta $\beta$ & 0.99448 & \\
Momentum compaction $\alpha$ & $2.839 \times 10^{-3}$ & \\
Revolution period $T_{0}$ & $11.13 \quad \mu \mathrm{s}$ \\
Revolution frequency $f_{0}$ & $0.0898 \mathrm{MHz}$ \\
Synchrotron tune at $V=65 \mathrm{kV}$ & $5.177 \times 10^{-4}$ & \\
Bucket width $t_{b}$ & 397.64 & $\mathrm{~ns}$ \\
\hline
\end{tabular}

Synchrotron oscillations occur whenever the bunch is not at the center of the rf bucket, or there are phase and energy mismatches. In the capture of an unbunched beam, we do not care so much about rf phase mismatch. Only energy mismatch is important. This occurs when the rf frequency is not exactly the $\mathrm{rf}$ harmonic $h=28$ times the revolution frequency of the on-energy beam. If the amplitude of the subsequent synchrotron oscillation is $\Delta \tau=15 \mathrm{~ns}$, the momentum mismatch is

$$
\frac{\Delta p}{p}=\frac{2 \pi \beta^{2} \omega_{s} \Delta \tau}{|\eta| T_{0}}=5.358 \times 10^{-4}
$$

or $\Delta E / E=5.299 \times 10^{-4}$.

A change of rf frequency by $\Delta f_{\mathrm{rf}}$ implies a change of on orbit length by

$$
\Delta C=-\frac{C^{2}}{\beta c h} \Delta f_{\mathrm{rf}}
$$

or

$$
\frac{\Delta C}{C}=-\frac{T_{0}}{h} \Delta f_{\mathrm{rf}}
$$


This new orbit corresponding to the shifted rf frequency differs from the unshifted one by the momentum off-set $\Delta p / p$, where

$$
\frac{\Delta C}{C}=\alpha \frac{\Delta p}{p}=-T_{0} h \Delta f_{\mathrm{rf}},
$$

with $\alpha$ being the momentum compaction factor. Thus the synchrotron oscillation amplitude of $\Delta \tau=15 \mathrm{~ns}$ implies an off-momentum of $\Delta p / p=5.358 \times 10^{-4}$ from the on-momentum of the shifted rf frequency. The shifted rf frequency is therefore

$$
\Delta f_{\mathrm{rf}}=-\frac{\alpha h}{T_{0}} \frac{\Delta p}{p}
$$

and the corresponding fractional rf frequency shift required to produce the observed synchrotron oscillation is

$$
\frac{\Delta f_{\mathrm{rf}}}{f_{\mathrm{rf}}}=-\alpha \frac{\Delta p}{p}=-1.521 \times 10^{-6}
$$

The drift in rf frequency is

$$
\Delta f_{\mathrm{rf}}=-3.825 \mathrm{~Hz}
$$

and the change in rf wavelength or bucket width is

$$
\Delta t_{b}=-t_{b} \frac{\Delta f_{\mathrm{rf}}}{f_{\mathrm{rf}}}=6.049 \times 10^{-4} \mathrm{~ns} .
$$

\section{Sources of Mismatch}

One possibility of the mismatch of the beam to the capturing rf can be random fluctuation of the $2.5-\mathrm{MHz}$ rf frequency. The frequency of the $2.5-\mathrm{MHz} \mathrm{rf}$ is locked on to the frequency of the $53-\mathrm{MHz}$ rf, so that their ratio is exactly $28: 588$. The $53-\mathrm{MHz}$ rf gets its frequency fixed from the oscillator in the rf system. Even when the 53-MHz rf voltage is turned off after adiabatic debunching of the injected Booster batch, the 53- $\mathrm{MHz}$ clock still stays in the rf system. As a result, there is no reason for the $2.5-\mathrm{MHz}$ rf to drift at all. For verification, the sinusoidal voltage of the $2.5-\mathrm{MHz}$ rf was displayed as a mountain-range plot for the whole debunch-capture process. Since the capture portion takes $90 \mathrm{~ms}$, a drift of $3.83 \mathrm{~Hz}$ amounts to $3.83 \times 0.09=0.344 \mathrm{rf}$ cycles, which should be evident in the mountain-range plot. However, such drift was not observed at all. We can therefore conclude that there has not been any drift in the $2.5-\mathrm{MHz}$ rf frequency. 
Even when there is no drift in the $2.5-\mathrm{MHz}$ rf frequency, there can still be an energy mismatch. This mismatch comes from the mismatch of injected beam energy with the 53$\mathrm{MHz}$ rf frequency. Energy mismatch implies dipole synchrotron oscillations especially before the lowering of the 53-MHz rf, after phase mismatch has been corrected. This mismatch will lead to a debunched beam with more positive energy offset than negative energy offset, or vice versa. This will result in right-left asymmetry of the line density as adiabatic capture progresses and we did observe such asymmetry during our study.

Before debunching, the energy mismatch will lead to dipole synchrotron oscillations. For the momentum offset $\Delta p / p$, the amplitude of synchrotron oscillation is given by Eq. (1), or

$$
\Delta \tau=\frac{|\eta| T_{0}}{2 \pi \beta^{2} \omega_{s}} \frac{\Delta p}{p}
$$

Comparing with the synchrotron oscillation in the 2.5-MHz bucket, the scaling is $\sqrt{h V}$. Here for the $53-\mathrm{MHz} \mathrm{rf}, h=588$ and $V=550 \mathrm{kV}$ were used. Thus the oscillation amplitude translates into

$$
(\Delta \tau)_{53}=(\Delta \tau)_{2.5} \sqrt{\frac{28 \times 65}{588 \times 550}}=1.0 \mathrm{~ns}
$$

In our memory, we did see synchrotron oscillation with amplitude $\Delta \tau \approx 1$ ns in the $53-\mathrm{MHz}$ rf bucket before the debunching process. This explains why the synchrotron oscillation was observed during adiabatic capture. It is important to note that the oscillation amplitude was amplified by about $\sqrt{(h V)_{53} /(h V)_{2.5}}=13.3$ times.

\section{Right-Left Asymmetry}

At the beginning of the adiabatic capture, it is reasonable to predict that only those particles staying outside the initial bucket setup by the $2.5-\mathrm{MHz}$ rf will contribute to right-left asymmetry of the linear beam density. Those particles staying inside the initial bucket will be smeared out and contribute very little to the asymmetry. Our calculation shows an energy mismatch of $\Delta E / E=5.299 \times 10^{-4}$ with the rf frequency. This amounts to $4.736 \mathrm{MeV}$, which is a big number. This is because if the injected booster bunches are of emittance $0.1 \mathrm{eVs}$, the beam after perfect debunching will have energy offset of only $\pm 2.640 \mathrm{MeV}$. This implies that the energy offset of the whole beam will be completely positive, if the mismatch is in the positive-energy direction. The comparison of the beam with the initial $2.5-\mathrm{MHz}$ bucket is shown in Fig. 2, where the initial rf voltages are, respectively, $0.5 \mathrm{kV}, 1 \mathrm{kV}, 2 \mathrm{kV}, 3 \mathrm{kV}$, 


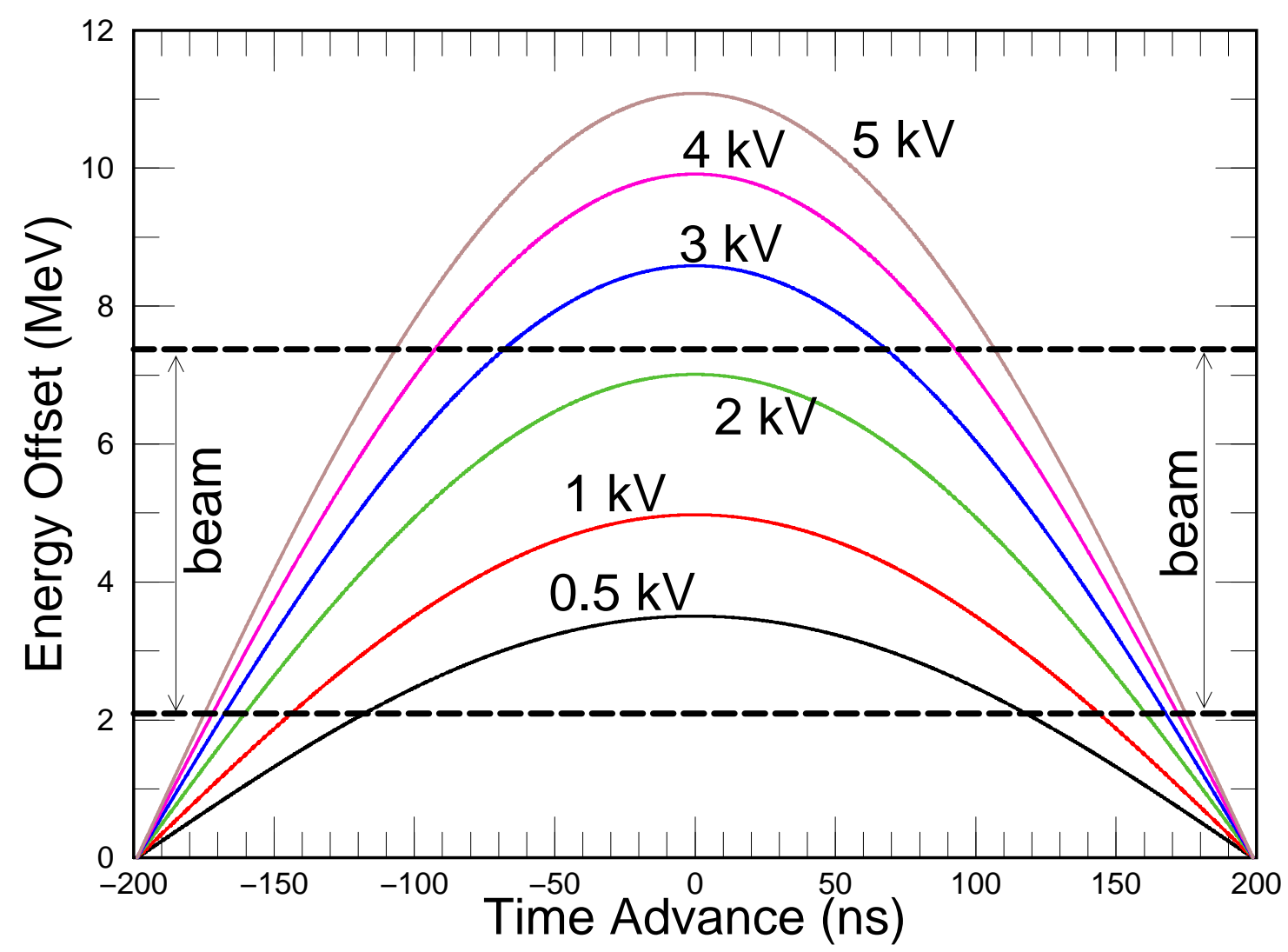

Figure 2: Positive parts of the bucket boundaries setup by initial $2.5 \mathrm{MHz}$ rf voltages $0.5 \mathrm{kV}, 1 \mathrm{kV}$, $2 \mathrm{kV}, 3 \mathrm{kV}, 4 \mathrm{kV}$, and $5 \mathrm{kV}$. The energy-mismatched beam lies between the two thick horizontal dashed lines.

$4 \mathrm{kV}$, and $5 \mathrm{kV}$. The beam is shown between the thick horizontal black dashed lines. Here, only the positive energy offset is displayed, because there is no beam in the negative energy offset. It is obvious that very small amount of the beam is enclosed inside the $0.5-\mathrm{kV} 2.5$ $\mathrm{MHz}$ bucket. The right-left symmetry is therefore expected to be large as is shown in Fig. 1. As the initial capture voltage increases, more and more beam particles are enclosed inside the initial rf bucket, and the right-left asymmetry is expected to decrease. This is verified by the observation shown in Fig. 3, where the initial capture voltages are, respectively, $2 \mathrm{kV}$ and $5 \mathrm{kV}$. 

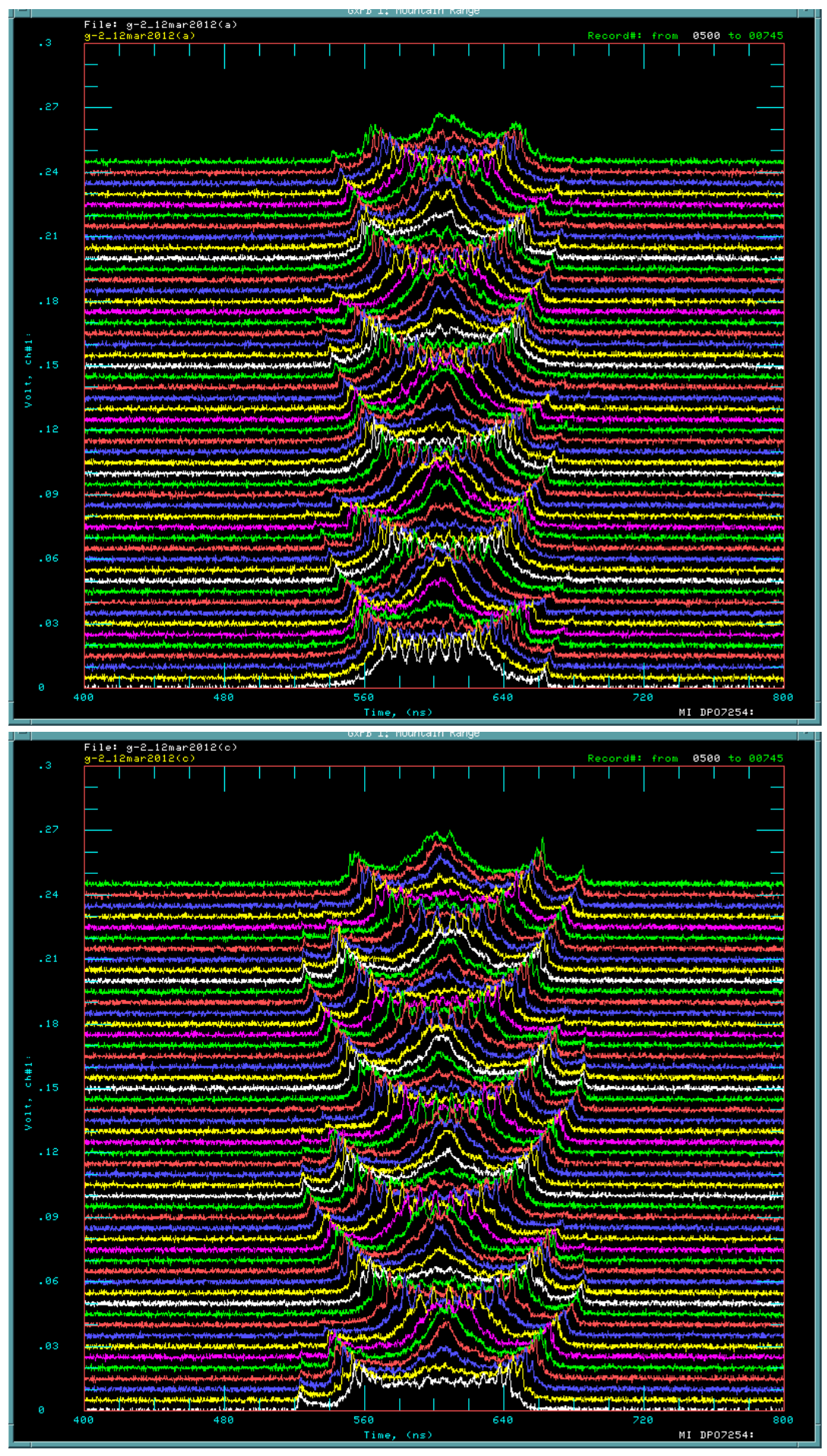

Figure 3: Top: The second of four bunches captured by $2.5 \mathrm{MHz}$ rf from 2.0 to $65 \mathrm{kV}$ in $90 \mathrm{~ms}$. Bottom: Same but with initial rf voltage $5.0 \mathrm{kV}$. Compare with Fig. 1, the right-left asymmetry has been reduced by very much as the initial capture voltage increases. 


\section{Conclusions}

We conclude that the right-left asymmetry and synchrotron oscillations observed during and after the 2.5-MHz adiabatic capture from an unbunched beam are due to energy mismatch of the beam with the $2.5-\mathrm{MHz}$ rf frequency. The source of this mismatch can come from two places:

1. The frequency drift of the $2.5-\mathrm{MHz}$ rf after the turning off of the $53-\mathrm{MHz}$ rf voltage can result in energy mismatch of the beam. An observed amplitude of 15-ns synchrotron oscillation can be explained by about $3.8-\mathrm{Hz}$ frequency drift of the $2.5-\mathrm{MHz}$ rf. However, the frequency of the $2.5-\mathrm{MHz}$ rf has been seen very stable in mountain-range plots during the whole debunch-capture process, so that this possibility can be ruled out.

2. The energy-mismatch can also come from the mismatch of the injected booster beam with the frequency of the $53-\mathrm{MHz}$ rf in the Main Injector. If there is no frequency drift of the 2.5-MHz rf, the 15-ns synchrotron oscillation amplitude observed during and after capture translates into a 1.0-ns synchrotron oscillation amplitude in the $53-\mathrm{MHz}$ rf bucket. Such amount of synchrotron oscillation had been observed.

A synchrotron oscillation in the $53-\mathrm{MHz}$ rf bucket is magnified in the 2.5-MHz rf bucket.

This magnification scales inversely with $\sqrt{h V}$, where $h$ is the rf harmonic and $V$ the rf voltage of the respective rf systems. In our example, this magnification has been 13.3-fold. Thus the energy mismatch must be carefully corrected in the $53-\mathrm{MHz}$ bucket in order to suppress synchrotron oscillations during and after the $2.5-\mathrm{MHz}$ capture. 